\title{
Peristaltic pumping through porous medium in presence of electric double layer
}

\author{
Ashu Yadav $^{1^{*}}$, Shashi Bhushan ${ }^{2}$, and Dharmendra Tripathi $^{2}$ \\ ${ }^{1}$ Automobile Engineering Department, Faculty of Engineering, Manipal University, Jaipur-303007, India \\ ${ }^{2}$ Mechanical Engineering Department, Faculty of Engineering, Manipal University, Jaipur-303007, India
}

\begin{abstract}
The present paper investigates the peristaltic flow of Newtonian fluids through the porous medium. The effects of electroosmosis mechanism on peristaltic pumping are also considered. An analytical solution is obtained under lubrication approach. Poisson Boltzmann equations are also simplified using Debye linearization. The effects of permeability parameter, electrical double layer thickness and electro-osmotic parameter on the flow characteristics, pressure distribution and shear stress distributions are computed. Numerical computations reveal that electroosmosis and permeability play vital role in peristaltic pumping. The findings of present study may be applicable in biomedical engineering and chemical engineering where peristaltic micropumps may be designed.
\end{abstract}

\section{Introduction}

Mathematical modeling of various real systems is governed by navier-stokes equations in various emerging field of Fluid dynamics. Understanding the basic transport mechanisms in biological systems are not as easy like engineering applications, because in-vivo experiments are not easy to perform. The concepts of modern bio-fluid mechanics can be applied to many systems in the human body. Gastrointestinal tract is an interesting part of the human physiological system that has many physical processes coupled with fluid dynamics. The physiology of the intestinal movements was investigated for the first time, confined to the small intestine of the dog, by Bayliss and Starling[1]. In the literature, the transport mechanism of chyme in intestines presets a special challenges to bio engineers Lew H. et al. [2] developed a mathematical model of peristalsis involved in small intestines based on low Reynolds number flow of a Newtonian fluid in a circular cylindrical tube. This idea was extended by many researchers to reflect the non-linear behavior of the chyme. Srivastava and Srivastava [3] studied fluid dynamic applications of vas deferens and small intestine in a uniform and non-uniform tube under the consideration of a non-Newtonian (Power law) fluid model.

Flow of fluids in channels under the effect of an electrical field is a key area in both medical engineering and energy sciences. Both electro-hydrodynamics and electro-kinetics (electro-osmotic transport) [4-6] are important aspects of this arena. The latter plays a prominent role in micro- and nano-channel systems in ionic flow delivery [7-9], petro-chemical sampling in low-permeability reservoirs [10], geotechnical remediation and field-based stabilization of geological masses [11]. Electrokinetics can be very successfully utilized to transform mechanical energy into electrical energy, to regulate flow delivery, achieve specified mass transfer rates etc. enhanced. Electro-osmotic flows in porous media are also of significance and arise in both biological systems and industrial systems and have been shown to markedly influence transport rates [12]. The classical approach to simulating transport in porous media is the Darcy model which is valid for viscousdominated, low Reynolds number flows. It has been implemented extensively in electrokinetic modelling for porous media and such studied provide a very important compliment to laboratory-based investigations [13]. Gupta et al. [14] analysed the electrokinetic flow in porous media micro-channels with Stern layer effects, computing expressions for surface charge density, electrical conductivity, and electroosmotic coupling coefficient for various porous structures and physicochemical boundary conditions

After reviewing the above literature, the present study aims to analyze the peristaltic flow of Newtonian fluids through porous medium in presence of electroosmosis. The influences of pertinent physical parameters on velocity, pressure and wall shear stress are examined. The present model can further be explored for various model of non- Newtonian fluids which are more relevant in biological environments.

\section{Mathematical model}

The geometric model for peristaltic flow through a finite length $(L)$ porous medium is depicted in Fig.1, and mathematically expressed as:

\footnotetext{
Corresponding author: ashu.yadav@jaipur.manipal.edu
} 


$$
\bar{h}(\bar{x}, \bar{t})=a+b \sin \frac{2 \pi}{\lambda}(\bar{x}-c \bar{t}),
$$

where $a, b, \lambda, \bar{x}, c, \bar{t}, L$ are the half width of the channel, amplitude of wave, wavelength, axial coordinate, wave velocity, time and channel length.

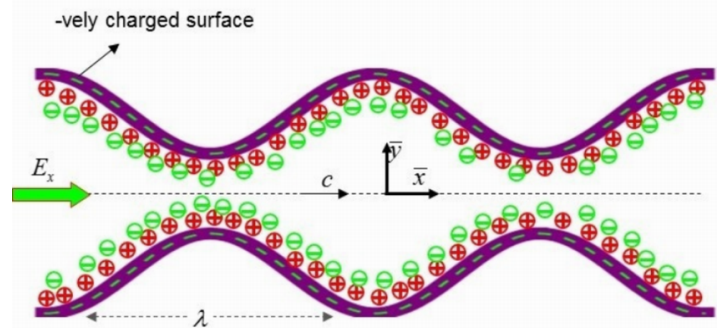

Fig. 1. Geometry for peristatic pumping through porous medium in presence of EDL.

The governing equations for two dimensional unsteady peristaltic flow of Newtonian fluids through porous medium in presence of EDL are expressed as:

$$
\begin{gathered}
\frac{\partial \bar{u}}{\partial \bar{x}}+\frac{\partial \bar{v}}{\partial \bar{y}}=0 \\
\rho\left(\frac{\partial \bar{u}}{\partial \bar{t}}+\bar{u} \frac{\partial \bar{u}}{\partial \bar{x}}+\bar{v} \frac{\partial \bar{u}}{\partial \bar{y}}\right)=-\frac{\partial \bar{p}}{\partial \bar{x}}+\mu\left(\frac{\partial^{2} \bar{u}}{\partial \bar{x}^{2}}+\frac{\partial^{2} \bar{u}}{\partial \bar{y}^{2}}\right)-\mu \frac{\bar{u}}{\bar{k}}+\rho_{e} \mathrm{E}_{x}, \\
\rho\left(\frac{\partial \bar{v}}{\partial \bar{t}}+\bar{u} \frac{\partial \bar{v}}{\partial \bar{x}}+\bar{v} \frac{\partial \bar{v}}{\partial \bar{y}}\right)=-\frac{\partial \bar{p}}{\partial \bar{y}}+\mu\left(\frac{\partial^{2} \bar{v}}{\partial \bar{y}^{2}}+\frac{\partial^{2} \bar{v}}{\partial \bar{y}^{2}}\right)-\mu \frac{\bar{v}}{\bar{k}},
\end{gathered}
$$

where $\rho, \bar{u}, \bar{v}, \bar{p}, \mu, \bar{k}$ and $E_{x}$ denote the fluid density, axial velocity, transverse velocity, pressure, fluid viscosity, the permeability of the porous medium and electrokinetic body force.

The Poisson's equation is defined as :

$$
\nabla^{2} \phi=-\frac{\bar{\rho}_{e}}{\varepsilon},
$$

in which $\rho_{e}$ is the density of the total ionic charge and $\varepsilon$ is the permittivity. The Boltzmann equation is expressed as :

$$
n^{ \pm}=n_{0} \operatorname{Exp}\left[ \pm \frac{e z \bar{\Phi}}{K_{B} T}\right]
$$

where $n_{0}$ represents concentration of ions at the bulk, which is independent of surface electrochemistry, $e$ is the electronic charge, is the charge balance, $K_{B}$ is the Boltzmann constant, and $T$ is the average temperature of the electrolytic solution.

Employing the following boundary conditions as ;

$$
\begin{aligned}
& x=\frac{\bar{x}}{\lambda}, y=\frac{\bar{y}}{a}, t=\frac{c \bar{t}}{\lambda}, u=\frac{\bar{u}}{c}, v=\frac{\bar{v}}{c \delta}, \delta=\frac{a}{\lambda}, h=\frac{\bar{h}}{a}, \\
& \phi=\frac{b}{a}, p=\frac{\bar{p} a^{2}}{\mu c \lambda}, \operatorname{Re}=\frac{\rho c a}{\mu}, k=\frac{\bar{k}}{a^{2}}, \Phi=\frac{\Phi}{\zeta},
\end{aligned}
$$

where $\delta$ is wave number, $\operatorname{Re}$ is the Reynolds number, $\zeta$ is the zeta potential, Applying long wavelength and low Reynolds number approximations, the above Eqs. (2) - (4) reduce to

$$
\begin{gathered}
\frac{\partial u}{\partial x}+\frac{\partial v}{\partial y}=0, \\
\frac{\partial p}{\partial x}=\frac{\partial^{2} u}{\partial y^{2}}-\frac{u}{k}+m^{2} U_{H S} \phi,
\end{gathered}
$$

$$
\frac{\partial p}{\partial y}=0
$$

where $m=a e z \sqrt{\frac{2 n_{0}}{\varepsilon K_{B} T}}$, is known as the electroosmotic parameter and $U_{H S}=-\frac{E_{x} \varepsilon \zeta}{\mu c}$ is the maximum electroosmotic velocity. Applying Debye-Hückel linearization approximation, Poisson-Boltzmann equation reduces to :

$$
\frac{\partial^{2} \Phi}{\partial y^{2}}=m^{2} \Phi
$$

The boundary conditions are :

$$
\begin{aligned}
& \left.\frac{\partial \Phi}{\partial y}\right|_{y=0}=0,\left.\Phi\right|_{y=h}=1,\left.\frac{\partial u}{\partial y}\right|_{y=0}=0,\left.u\right|_{y=h}=0,\left.v\right|_{y=0}=0, \\
& \left.v\right|_{y=h}=\left.\frac{\partial h}{\partial t} p\right|_{x=0}=p_{0} \text { and }\left.p\right|_{x=L}=p_{L}
\end{aligned}
$$

The solution of above governing equations subjected to boundary conditions are

$$
\begin{gathered}
\Phi=\frac{\cosh (m y)}{\cosh (m h)}, \\
u=k \frac{\partial p}{\partial x}\left\{\frac{\cosh \left(\frac{y}{\sqrt{k}}\right)}{\cosh \left(\frac{h}{\sqrt{k}}\right)}-1\right\}+\frac{m^{2} U_{H S}}{m^{2}-\frac{1}{k}}\left\{\frac{\cosh \left(\frac{y}{\sqrt{k}}\right)}{\cosh \left(\frac{h}{\sqrt{k}}\right)}-\frac{\cosh (m y)}{\cosh (m h)}\right\}
\end{gathered}
$$

Furthermore, utilizing Eq. (13) with continuity equation, and boundary condition (11), the pressure gradient emerges as :

$$
\frac{\partial p}{\partial x}=\frac{1}{k^{3 / 2}\left(\frac{h}{\sqrt{k}}-\tanh \left(\frac{h}{\sqrt{k}}\right)\right)}\left[G_{0}(t)+\int \frac{\partial h}{\partial t} d x-\frac{m^{2} U_{H S}}{m^{2}-\frac{1}{k}}\left\{\begin{array}{c}
\sqrt{k} \tanh \left(\frac{h}{\sqrt{k}}\right) \\
-\frac{\tanh (m h)}{m}
\end{array}\right\}\right]
$$

The pressure difference can be obtained as :

$$
\Delta p=p(\mathrm{x}, t)-p(0, t) \int_{0}^{x} \frac{\partial p}{\partial s} d s,
$$

where $G_{0}(t)$ is expressed as:

$$
G_{0}(t)=\frac{\left(p_{l}-p_{0}\right)-\int_{0}^{L} \frac{1}{k^{3 / 2}\left(\frac{h}{\sqrt{k}}-\tanh \left(\frac{h}{\sqrt{k}}\right)\right.}\left[\int \frac{\partial h}{\partial t} d x-\frac{m^{2} U_{H S}}{m^{2}-\frac{1}{k}}\left\{-\frac{\sqrt{k} \tanh \left(\frac{h}{\sqrt{k}}\right)}{m}\right)\right] d x}{\int_{0}^{L} \frac{1}{k^{3 / 2}\left(\frac{h}{\sqrt{k}}-\tanh \left(\frac{h}{\sqrt{k}}\right)\right)} d x}
$$

The local wall shear stress is derived as :

$$
\tau_{w}=\left.\frac{\partial u}{\partial y}\right|_{y=h}=\sqrt{k} \tanh \left(\frac{h}{\sqrt{k}}\right) \frac{\partial p}{\partial x}-\frac{m^{2} U_{H S}}{m^{2}-\frac{1}{k}}\left\{\frac{\tanh \left(\frac{h}{\sqrt{k}}\right)}{\sqrt{k}}-m \tanh (m h)\right\}
$$

\section{Results and Discussion}

Numerical computations for axial velocity, pressure distribution and local wall shear stress have performed under the effects of debye length, electroosmotic velocity and permebility parameter. To visualize the effects of physical parameters, Figs. 2-4 are illustrated.

Figs. 2a-c illustrate the evolution of axial velocity $(u)$ with EDL thickness $(m)$, external electric field $\left(U_{H S}\right)$ and permeability parameter $(k)$ respectively. Fig. 2a shows the effect of EDL thickness on velocity profile, 
and it is noted that the axial velocity increases with increasing the EDL thickness. Fig. 2b depicts the effect of external electric field on velocity and it is noted that axial velocity increases with increasing external electric field. Fig. 2c depicts the effect of permeability parameter on velocity profile and it is noted that axial velocity increases with increasing permeability parameter.

(a)

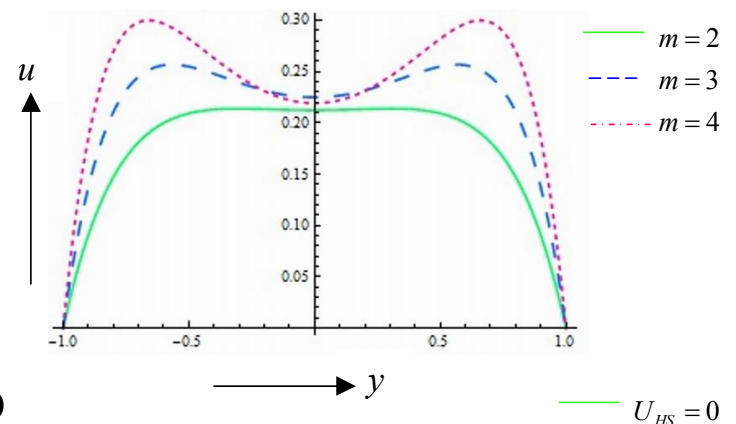

(b)

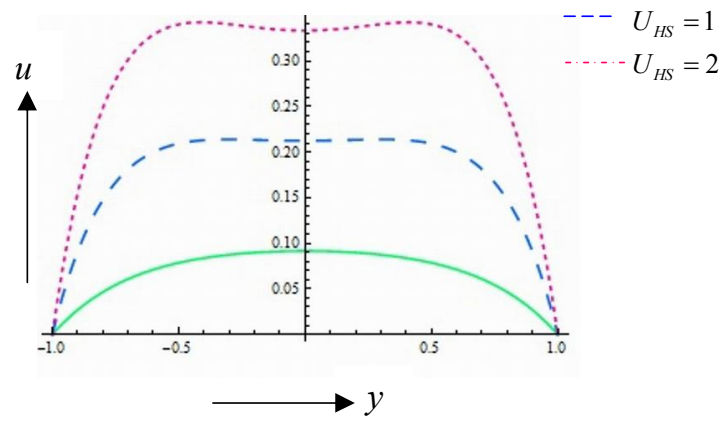

(c)

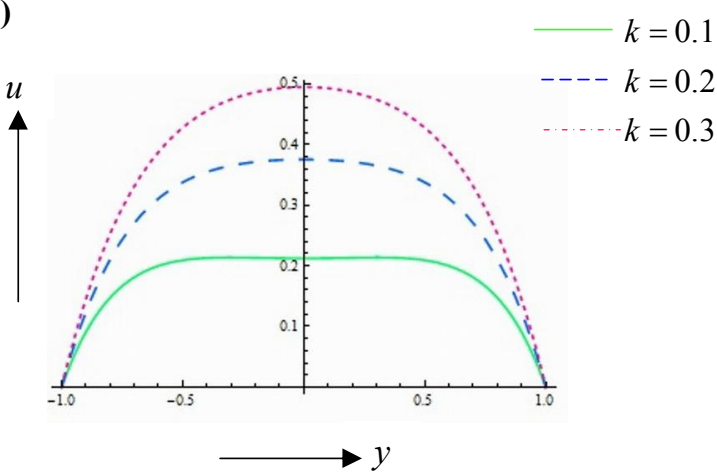

Fig. 2. Velocity profile (axial velocity vs. transverse coordinate) at $\varphi=0.5, x=0$ and (a) $U_{H S}=1, k=0.1$ (b) $m=2, k=0.1$ (c) $U_{H S}=1, m=2$.

Figs. 3 a-c present the pressure difference across the channel with EDL thickness $(m)$, external electric field $\left(U_{H S}\right)$ and permeability parameter $(k)$ respectively. It is observed that pressure difference is highest at the wall contraction and its magnitude increases with increasing the value of EDL thickness and external electric field while it decreases with increasing permeability parameter. The effect of external electric field is quite significant as compared with effect of EDL thickness. (a)
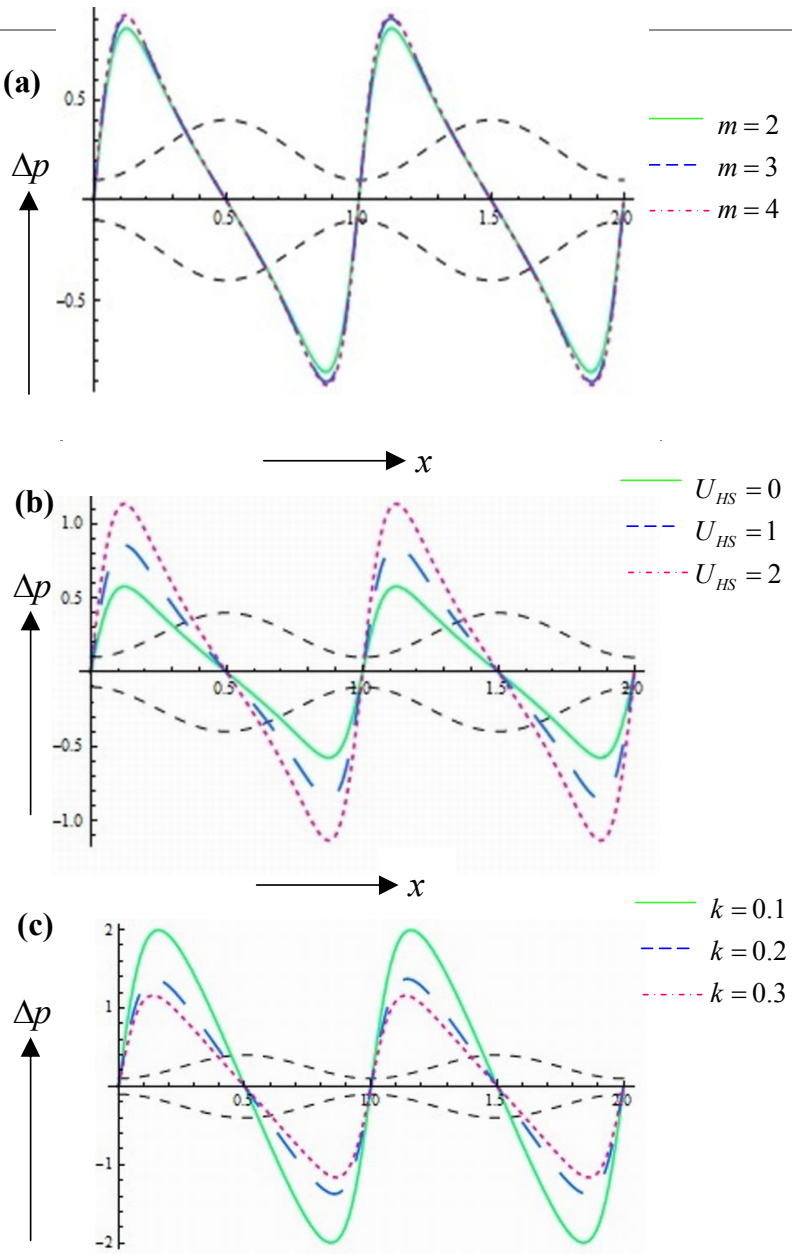

Fig. 3. Pressure distribution a $x$ he length of channel at $\varphi=0.5, \mathrm{t}=0.25, p_{L}=p_{0}=0$ and (a) $U_{H S}=1, k=0.1$

(b) $m=2, k=0.1$ (c) $U_{H S}=1, m=2$.

Figs. 4 a-c depict the shear stress distribution along the channel length with EDL thickness $(m)$, external electric field $\left(U_{H S}\right)$ and permeability parameter $(k)$ respectively. The magnitude of shear stress is hightest near the contraction and minimum where channel is releaxed. Further it is observed that local wall shear stress increses with increasing the value of EDL thickness and external electric field, but permeability parameter has reverse effect on wall shear stress.

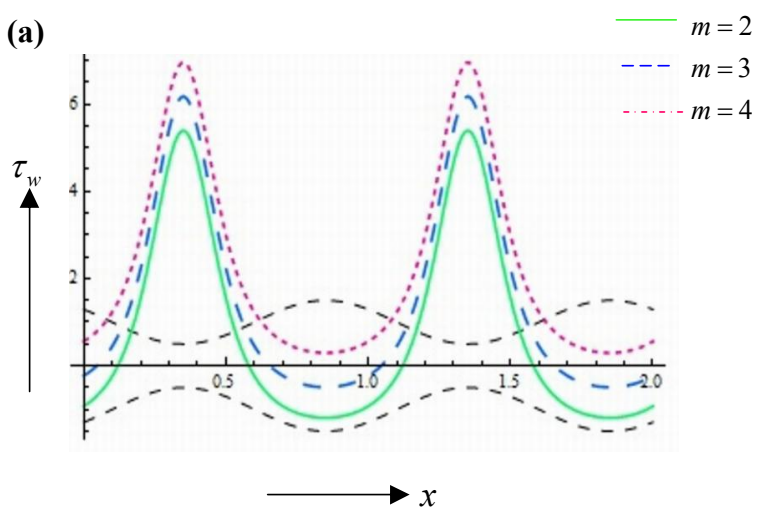


(b)

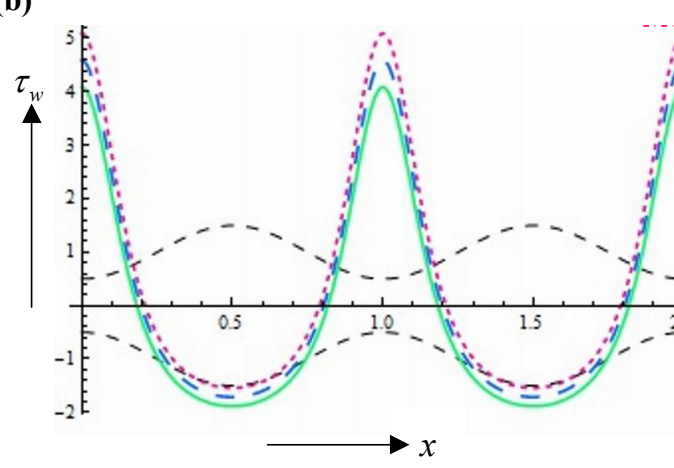

(c)

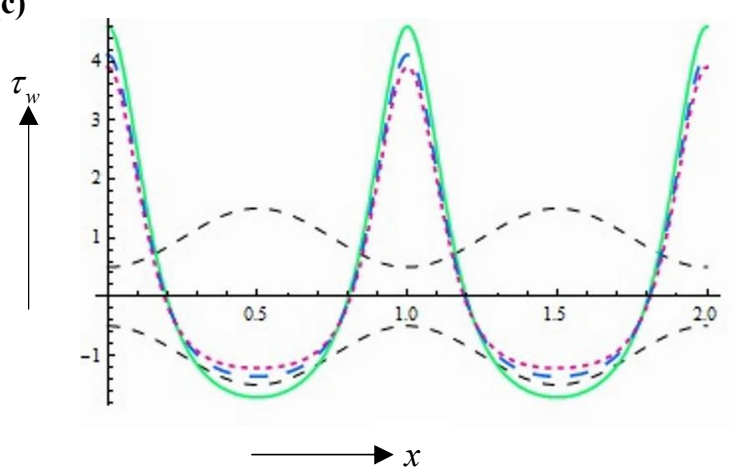

Fig. 4. Local wall shear stress along the length of channel at $t=0.25, \varphi=0.5$, (a) $U_{H S}=1, k=0.1$ (b) $m=1, k=0.1$ (c) $U_{H S}=1, m=1$

\section{Conclusions}

An analytical model is developed to study the peristaltic flow of Newtonian fluids through porous medium. The effects of permeability parameter, EDL thickness and external electric field on flow characteristics, pumping characteristics (i.e. pressure distribution) and wall shear distribution along the channel length are analyzed. It is noted that axial velocity strongly depends on the permeability, EDL thickness and external electric field. It is also concluded that peristaltic mechanisms can be controlled with applying the external electric field. The permeability of the medium resists the peristaltic flow process. That means if the medium is highly porous then peristaltic pumping can be maintained by external electric field. It is further noted that wall shear stress alters with electroosmosis and permeability. The finding of present model can be helpful in biomedical and industrial applications.

\section{References}

1 W. Bayliss and E. Starling, The Journal of physiology, 24, pp. 99-143, (1899).

2 H. Lew, Y. Fung, and C. Lowenstein, Journal of Biomechanics, 4, pp. 297-315, (1971).
L. Srivastava and V. Srivastava, Annals of biomedical engineering, 13, pp. 137-153, (1985).

C.-O. Ng and Q. Zhou, Fluid Dynamics Research, 44, p. 055507, (2012).

5 Y. Kang, K. Ooi, C. Yang, and T. Wong, Journal of Micromechanics and Microengineering, 15, p. 301, (2004).

6 G. D. Ngoma and F. Erchiqui, Journal of Micromechanics and Microengineering, 16, p. 83, (2005).

7 K. Kim, H. S. Kwak, and T.-H. Song, Fluid Dynamics Research, 43, p. 041401, (2011).

8 K.-D. Huang and R.-J. Yang, Nanotechnology, 18, p. 115701, (2007).

Y. Ren and D. Stein, Nanotechnology, 19, p. 195707, (2008).

10 W. Zhang, J. Yao, Y. Gao, Q. Zhang, and H. Sun, Journal of Petroleum Science and Engineering, 134, pp. 150-157, (2015).

11 R. Azzam and W. Oey, Transport in Porous Media, 42, pp. 293-314, (2001).

12 D. B. Pengra, L. Shi, S. X. Li, and P.-Z. Wong, "Experimental study of electrokinetics in porous media," in MRS Proceedings, p. 201, 1994.

13 R. S. Maier, E. Nybo, J. D. Seymour, and S. L. Codd, Transport in Porous Media, 113, pp. 6789, (2016).

14 A. Gupta, D. Coelho, and P. Adler, Journal of colloid and interface science, 316, pp. 140-159, (2007). 